\section{Seawater carbon measurement}

SIR - Ogawa and Ogura ${ }^{1}$ compared high-temperature catalytic oxidation (HTCO) and wet chemical oxidation (WCO) methods for measuring dissolved organic carbon (DOC) in sea water. They concluded that both methods gave similar results, although concentrations derived from HTCO were 15-25\% higher for bulk ocean water, and 30$40 \%$ higher for two low relative molecular mass (less than 1,000) DOC fractions. These results are in contrast to an earlier report ${ }^{2}$ that seawater DOC includes a substantial high molecular mass fraction which can be measured only by HTCO methods and is present in concentrations sufficient to more than double the size of this already immense organic carbon pool ${ }^{3}$. On analysis of Ogawa and Ogura's data, we find evidence that their HTCO blank could largely explain much of the difference between the reported HTCO and WCO measurements as well as their evidence for an apparent WCO-resistant low molecular mass fraction.

The experimental design used by Ogawa and Ogura compared HTCO and WCO measurements of unconcentrated DOC in bulk ocean water and low molecular mass $(>1 \mathrm{k})$ fractions as well as of concentrated high molecular mass $(>10 \mathrm{~K}$ and $>1 \mathrm{~K})$ DOC in ultrafiltered samples. The DOC values for the latter samples were reported in terms of initial concentrations after dividing the measured final value by the concentration factor. The authors measured total blanks for both procedures using doubledistilled water, obtaining means of 10.5 and $3.8 \mu \mathrm{M}$ for HTCO and WCO, respectively. They did not correct their reported DOC values for these backgrounds, in part because it was difficult to determine whether the blank signals were derived from the instruments or the distilled water.

A correlation plot of data from Ogawa and Ogura's Tables 1 and 2 shows that the HTCO- and WCO-based measurements of DOC in their unconcentrated bulk seawater and low molecular mass fractions correlate highly $\left(r^{2}=0.979\right.$, $\mathrm{n}=13$ ). The best-fit line, however, intercepts the HTCO axis at $15.0 \pm 3.2 \mu \mathrm{M}$ (see figure). This intercept indicates that the HTCO method had an average blank approximately $15 \mu \mathrm{M}$ higher than for the WCO method, a somewhat greater difference than indicated by the distilled water blanks. Blanks of this magnitude and greater have been observed for various commercial HTCO analysers ${ }^{4}$. It is significant that if the two analyses had equal blanks, but the HTCO instrument measured a constant fraction of the total DOC not determinable by WCO, the correlation line would extrapolate to the origin.

The larger HTCO blank most affects analyses of more dilute bulk and $<1 \mathrm{~K}$ samples for which the $15 \mu \mathrm{M}$ higher background accounts for $12-34 \%$ of the total measured values. Well over half of the differences in the raw WCO/HTCO ratios listed by Ogawa and Ogura in their Table 1 apparently

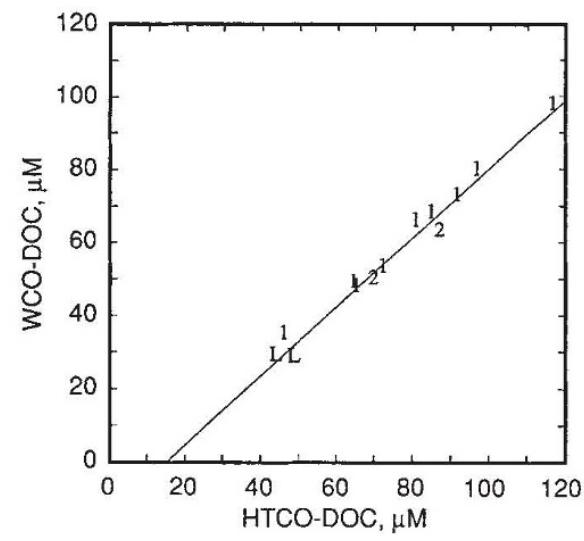

Correlation between wet chemical oxidation (WCO) and high temperature catalytic oxidation (HTCO) measurements ${ }^{1}$ of unconcentrated dissolved organic carbon (DOC) in sea waters of the western North Pacific. Data for bulk DOC from Tables 1 and 2 of ref. 1 are indicated by these numbers and $L$ indicates concentrations measured for the lowmolecular-mass $(<1 \mathrm{~K})$ fractions in Table 2 of ref. 1 . The plotted line is the best leastsquares fit to the data.

result from the higher HTCO blank, as opposed to a greater response of the HTCO versus the WCO instrument. In contrast, the HTCO blank is relatively inconsequential in measurements of the DOC content of the high molecular mass samples, most of which were concentrated to the extent (usually 20 times) that the offset in the HTCO blank corresponds to less than $2 \%$ of the total signal.

This interpretation strengthens Ogawa and Ogura's principal finding that the HTCO and WCO methods they compared measure similar concentrations of DOC for various sea waters. Evidence for a substantial fraction of WCOresistant $\mathrm{DOC}$ in the low molecular mass fraction of these water samples, however, is correspondingly weakened. Our re-evaluation demonstrates that instrument blanks and data treatments must be carefully considered when measuring and interpreting DOC con-

\footnotetext{
1. Ogawa, H. \& Ogura, N. Nature 356, 696-698 (1992).

2. Sugimura, Y. \& Suzuki, Y. Mar. Chem. 24, 105-131 (1988).

3. Toggweiler, J. R. Nature 356, 665-666 (1992)

4. Benner, R. \& Strom, M. Mar. Chem. (in the press).
}

centrations. Wide availability and use of DOC-free water would make determinations of DOC analyser blanks much easier.

John I. Hedges

Brian A. Bergamaschi

School of Oceanography WB-10,

University of Washington,

Seattle,

Washington 98195, USA

OGAWA AND OGURA REPLY - We agree with Hedges and Bergamaschi that the differences between the measurements could be smaller if the HTCO results are corrected for the system blank. We think that uncertainty in measuring DOC in sea water results mainly from the differences in the scale of blanks with the HTCO instruments and how the results are corrected for the blanks.

Additionally, we wish to emphasize that both low and constant blanks stably obtained throughout the sample measurements are important when the results are corrected for the blanks. As indicated by Hedges and Bergamaschi, the blank values for our HTCO instrument (Shimadzu TOC-5000) seem to be lower than those reported elsewhere, but we do not believe that our instrument is the only type for which this is the case.

We found that elevated blanks could be obtained from deionized/distilled water and that they could be reduced and fixed to some extent by repeated injections of the deionized/distilled water. We conducted the DOC measurements for standard and sample solutions when we confirmed the low and constant blanks after this conditioning process. As shown in Hedges and Bergamaschi's figure, a strong association with an intercept given between the two methods supports the consistency of system blanks for our HTCO analysis.

H. Ogawa

N. Ogura

Department of Environmental Science and Conservation,

Faculty of Agriculture,

Tokyo University of Agriculture and Technology,

3-5-8 Saiwaicho, Fuchu-shi, Tokyo 183, Japan

\section{Scientific Correspondence}

Scientific Correspondence is a relatively informal section of Nature in which matters of general scientific interest, not necessarily those arising from papers appearing in Nature, are published. Because there is space to print only a small proportion of the letters received, priority is usually given according to general interest and topicality, to contributions of fewer than 500 words, and to contributions using simple language. 